\title{
BMJ Open Updated systematic review and meta- analysis of studies examining the relationship between reported racism and health and well-being for children and youth: a protocol
}

Naomi Priest (D) ,1,2 Kate Doery, ${ }^{1,2}$ Mandy Truong, ${ }^{3}$ Shuaijun Guo, ${ }^{2,4}$ Ryan Perry, ${ }^{1,2}$ Brigid Trenerry, ${ }^{5}$ Saffron Karlsen (D) , ${ }^{6}$ Yvonne Kelly (D) , ${ }^{7}$ Yin Paradies ${ }^{8}$

To cite: Priest N, Doery K, Truong M, et al. Updated systematic review and metaanalysis of studies examining the relationship between reported racism and health and well-being for children and youth: a protocol. BMJ Open 2021;11:e043722. doi:10.1136/ bmjopen-2020-043722

- Prepublication history and additional supplemental material for this paper are available online. To view these files, please visit the journal online (http://dx.doi.org/10.1136/ bmjopen-2020-043722).

Received 12 August 2020 Accepted 18 May 2021

Check for updates

(c) Author(s) (or their employer(s)) 2021. Re-use permitted under CC BY-NC. No commercial re-use. See rights and permissions. Published by BMJ.

For numbered affiliations see end of article.

Correspondence to

Dr Naomi Priest;

Naomi.Priest@anu.edu.au

\section{ABSTRACT}

Introduction Racism is a critical determinant of health and health inequities for children and youth. This protocol aims to update the first systematic review conducted by Priest et al (2013), including a meta-analysis of findings. Based on previous empirical data, it is anticipated that child and youth health will be negatively impacted by racism. Findings from this review will provide updated evidence of effect sizes across outcomes and identify moderators and mediators of relationships between racism and health.

Methods and analysis This systematic review and metaanalysis will include studies that examine associations between experiences of racism and racial discrimination with health outcomes of children and youth aged 0-24 years. Exposure measures include self-reported or proxy reported systemic, interpersonal and intrapersonal racism. Outcome measures include general health and well-being, physical health, mental health, biological markers, healthcare utilisation and health behaviours. A comprehensive search of studies from the earliest time available to 0ctober 2020 will be conducted. A random effects meta-analysis will examine the average effect of racism on a range of health outcomes. Study-level moderation will test the difference in effect sizes with regard to various sample and exposure characteristics. This review has been registered with the International Prospective Register of Systematic Reviews.

Ethics and dissemination This review will provide evidence for future research within the field and help to support policy and practice development. Results will be widely disseminated to both academic and non-academic audiences through peer-review publications, community summaries and presentations to research, policy, practice and community audiences.

PROSPERO registration number CRD42020184055.

\section{INTRODUCTION}

Racism and racial discrimination are widely recognised as critical determinants of health and health inequities for children and youth across populations and
Strengths and limitations of this study

- This protocol aims to update the international review of racism and child health conducted by Priest et al (2013).

- The updated protocol now extends to include study participants aged 0-24 years to recognise the importance of youth development.

- This protocol includes a broad search strategy that aims to capture varied exposure measures of racism and racial discrimination as well as health and wellbeing outcomes.

- This protocol describes a meta-analysis to be conducted exploring relationships between racism and health among children and youth.

- This systematic review has a bias towards papers published in English, meaning that studies not published in English will not be included in this review.

contexts. ${ }^{1-3}$ Racism is a system of oppression that categorises and stratifies social groups into 'races', devalues and disadvantages those considered inferior and differentially allocates to them valued societal resources and opportunities. ${ }^{45}$ Racism is expressed across multiple levels, including systemic or structural racism, embedded within society; interpersonal racism between two or more individuals and includes racially motivated assaults or abuse and intrapersonal racism, whereby people take on negative stereotypes and beliefs about themselves. ${ }^{6-8}$ Racism and racial discrimination operates in many forms including direct and vicarious racism (secondhand racism), whereby individuals experience racism on a secondary level, witnessing or being informed of family, friends and strangers experiencing racism. ${ }^{10}$ Racism profoundly and perniciously impacts indigenous and racialised 
peoples throughout the world, including children and youth, for whom racism and racial discrimination is a major burden and influence on their health and development throughout life. ${ }^{1211}$

Research on racism and health has predominantly focused on interpersonal experiences, with considerable evidence documenting negative health effects across multiple outcomes, including physical and mental health outcomes. ${ }^{512} 13$ However, most of this evidence focused on adults, with far less research conducted among children and youth.

Priest et $a l^{\mathrm{l}}$ conducted the first international systematic review of quantitative studies on reported racial discrimination and the health and well-being of children and youth, including 121 studies. Since this report was published in 2013, the contribution of racism as a social determinant of health and well-being among children and youth has received growing attention. ${ }^{3}$ There is increasing evidence of the impact of racism on pathophysiological processes (eg, allostatic load and stress neurobiology) and biological markers (eg, C reactive protein and cortisol) ${ }^{14}$ as well as on health behaviours such as sleep ${ }^{15-17}$ among children and youth. The American Academy of Pediatrics recently issued a policy statement highlighting the impact that racism has on young people's health and health inequities and that addressing racism needs to be an urgent priority. ${ }^{2}$

A recent review of vicarious racism and child health found 30 studies published up to May 2016 compared with 10 studies in the previous 2013 review (with studies searched up to November 2011). ${ }^{9}$ This represents a threefold increase in studies examining vicarious racism and child health in approximately $4 \frac{1}{2}$ years. Additionally, our original review found that two-thirds of the included studies were published between 2005 and 2012. ${ }^{1}$ In light of the growing research in the field, there is a need to review and reflect on the current evidence to inform future scholarship in this area.

This present systematic review and meta-analysis aims to update findings from the 2013 review conducted by Priest $e t a l .{ }^{1}$ An updated systematic review is necessary to include new data, new methods and updated analysis. ${ }^{18}$ In this instance, an updated systematic review is necessary due to changing social policy and demographic contexts and new health priorities globally, as well as an increase in the number of recent publications in this area, including in different country and population contexts. The first systematic review identified that there were a limited number of longitudinal studies that have explored the health effects of racism on children and a need to expand research in this area, with a focus needed on the complex pathways to which child and youth health is impacted by experiences of racial discrimination. ${ }^{1}$ Priest $e t$ al called for an increase in high-quality longitudinal research using robust multidimensional measures of racial discrimination. ${ }^{1}$ As highlighted since this review was published in 2013, there has been a large increase in the amount of research being conducted in this field.
To answer our research question 'to what extent are experiences of racism associated with health and wellbeing outcomes among children and youth compared with those who experience no or less racism', we will use the previous review (Priest $e t a l^{1}$ ) as a guide, building on it and using an updated inclusion and exclusion strategy as well as expanding it to include a meta-analysis. As indicated by Garner $e t a l,{ }^{18}$ an updated systematic review can have an updated inclusion criteria while answering a similar question. This systematic review and meta-analysis aims to quantify the effects of racial discrimination on child and youth health, examine the key pathways by which racial discrimination influences these outcomes and identify potential moderators and mediators between racism and health. This review will provide key recommendations for future research and inform the development of effective evidence-based strategies for addressing racism and ameliorating its harmful effects.

\section{METHODS AND ANALYSIS}

This systematic review and meta-analysis will follow the guidelines of the Preferred Reporting Items for Systematic Reviews and Meta-Analysis (PRISMA) ${ }^{19}$ with the PRISMA Protocols ${ }^{20}$ checklist followed for the writing of this protocol (see online supplemental file 1). Progress on this systematic review and meta-analysis will be updated on the International Prospective Register of Systematic Reviews to maintain transparency.

\section{Inclusion criteria}

This report will include primary empirical studies that use quantitative methods including but not limited to cross-sectional; prospective and retrospective cohort; case-control designs; quasi-experimental studies and randomised control trials. Peer-reviewed journal articles (published or available as preprints) and dissertations/ theses will be included. We will also include grey literature such as published reports. Studies that do not report primary empirical associations between racism and child and youth health will not be included. Editorials and commentaries will not be included unless they report primary empirical data. According to the Population, Intervention, Comparison and Outcomes tool outlined in the Cochrane Handbook, ${ }^{21}$ we defined our population, intervention (exposure of interest) and specific outcome measures.

\section{Population}

Participants will include children and youth aged up to 24 years from any racial/ethnic/cultural groups. The age range of participants has been updated since the previous review (which included participants up to 18 years) to include children and youth as per the United Nations definition. ${ }^{22-24}$ This broader age range will allow for consideration of the health impacts of racism into late adolescence which is now considered by the Lancet 
Commission on Adolescent Health as extending to 24 years. ${ }^{24}$

\section{Exposure}

This review will extract childhood experiences of racism and racial discrimination exposures, synthesised across three main categories in the meta-analysis: systemic racism; interpersonal experiences including direct and vicarious or secondhand experiences (eg, witnessing or hearing about racism experienced by family, friends or a group an individual belongs to) and intrapersonal racism (eg, adapting racist attitudes and/or beliefs within their world views). Both personal and proxy reports (by parents or caregivers) of experiences of racism will be recorded, as will the source of racism (categorised as peers, teachers and community).

Terms used to determine the exposure include but are not limited to racism, discrimination, prejudice, harassment, bullying, stereotypes and unfair treatment where reason includes the victims' race/ethnicity/cultural background or proxy indicators such as migrant background, skin colour, language or accent. Although we recognise that religion is often highly racialised ${ }^{25}$ studies of religious discrimination will not be included as we consider this a related, but distinct form of discrimination. Religious discrimination is distinguished by being an assault on an individual or group's belief system and warrants independent investigation. ${ }^{26}$

There will be no restrictions placed on the timeframe of exposure to racism prior to the measurement. Retrospective adult population studies that report on childhood experiences of racism will be noted, but will not be included in our analysis.

\section{Outcome measures}

Studies will be considered if they measure health outcomes in children and youth. Health and well-being outcomes include measures of ill health and illness as well as positive health outcomes across physical, mental and behavioural domains. As guided by previous reviews and research, ${ }^{1} 1215$ 27-32 the following health and well-being outcomes will be included:

1. Pregnancy and birth outcomes (eg, premature birth, low birth weight).

2. General health and well-being, life satisfaction and quality of life.

3. Physical health (infectious disease and chronic conditions and markers, for example, body mass index, waist-hip ratio, blood pressure, metabolic and cardiovascular disease, overweight, obesity).

4. Negative mental health (eg, social and emotional difficulties, psychological distress, mental illness, suicide risk, self-harm, psychosis, antisocial behaviours including aggression and violence).

5. Positive mental health (eg, self-esteem, self-worth and resilience).

6. Health behaviours (eg, alcohol, tobacco, substance use) and sleep.
7. Healthcare utilisation, healthcare costs, satisfaction with child healthcare system (use of screening tests, maternal child healthcare, access to healthcare and treatment, adherence to treatment).

8. Biological markers (eg, inflammation and cardiometabolic markers).

\section{Exclusion criteria}

Studies reporting the effects of reported racism on other outcomes (eg, cognitive development, education, employment) will not be included. Studies that do not attribute discrimination experiences to race or ethnicity but only report generalised discrimination or unfair treatment without attribution will not be included. Studies published in a language other than English will not be included. Qualitative studies or studies only reporting the prevalence of racism without identifying associations with health and well-being outcomes will not be included.

\section{Data extraction and management}

\section{Search strategy}

The search strategy will be conducted in English and include studies from the earliest time available to October 2020. The search strategy will not be restricted to papers only published since the completion of the previous search strategy in 2011 as databases regularly back index studies and therefore some studies may have been missed by the original review. ${ }^{1}$ The search will be checked against the original search results to ensure that all studies that have been back indexed are also included.

The search will be conducted in the Ovid Medline, Ovid PsycInfo, PubMed, ERIC and ProQuest (for dissertation/ theses) databases. Reference lists of included studies will also be hand searched for additional relevant studies. The authors will also search Google Scholar to identify papers and reports citing the previous review and will search grey literature databases including Open Grey, OpenDOAR and New York Academy of Medicine using keywords from the search strategy.

The search will be performed using a string template combining search terms relevant to our study population, exposure and outcomes. The search strategy template has been developed in consultation with medical library staff using the previous search strategy by Priest $e a^{1}$ as a template. The search template to be used for Medline is included as online supplemental file 2 , which will be updated accordingly for each database.

\section{Selection of studies}

One member of the review team will conduct the initial search in the selected databases with the search results to be imported into Endnote X9. ${ }^{33}$ All titles and abstracts of studies identified in the search will be independently screened for eligibility for inclusion independently by two members of the review team using Covidence ${ }^{34}$ with any discrepancies resolved by the lead author. Duplicates and papers not in English will be deleted and noted in the PRISMA flowchart. ${ }^{19}$ Full-text studies will be assessed for 
final inclusion. Any discrepancies between members of the review team will be resolved by having a third member of the review team adjudicate the decision. Rationale for exclusion of studies will be noted throughout the screening process with a PRISMA flowchart ${ }^{19}$ being used to show the full selection process of studies.

\section{Data extraction}

Once the full-text studies have been identified, members of the review team will extract the data using Airtable. ${ }^{35}$ Two reviewers will independently extract data from each study, with inconsistencies and discrepancies resolved through discussion. Data from some studies may appear in multiple publications. If publications include unique combinations of exposure and outcome variables, they will be extracted as distinct data sets, meaning that one study may be included in the meta-analysis multiple times as different datasets due to its use of multiple measures of health or racism.

This review will examine the key characteristics of studies of reported racism and health and well-being among children and youth. Data to be extracted will include: authors; year of publication; study design (including sampling methods); definition of racism, exposure measure(s) (including tools/instruments and psychometric properties when applicable, method of administration including informant(s), content and time frames of exposure, targets and perpetrators, reactions/responses to racism and settings in which racism is experienced); health and well-being outcome measures; measures of racial/cultural/ethnic background; study location (country/region); place of residence (urban/ rural), sample size; participant demographics (age, racial/cultural backgrounds, gender, socioeconomic status, migration status); study findings; prevalence of self-reported racism including exposure characteristics; nature of associations between self-reported racism and health and well-being outcomes including subgroup analysis when reported (mean, SD, effect size); confounders, effect moderator and mediators of these associations and study quality/critical appraisal.

Effect sizes such as coefficients and $p$ values for each health outcome will be extracted. Both unadjusted and adjusted effect sizes will be extracted when available and covariates included in models recorded. Where an overall effect size is reported across a range of age groups, we will extract subgroup effect sizes when reported. In this case, only effect sizes for children and youth will be extracted.

Data including study characteristics, participant characteristics and exposure and outcome characteristics will be extracted to be included in the narrative synthesis but will not be included in the meta-analysis.

\section{Assessment of study quality and bias}

Studies included in the review will be critically appraised to determine the validity of the study's findings from the known literature and to provide readers with the ability to make an informed decision on the quality of these findings. Two members of the review team will independently rate studies selected for the meta-analysis using the Newcastle-Ottawa Scale, a widely used tool for evaluating the quality of non-randomised studies on a range of criteria. ${ }^{36}$ The Newcastle-Ottawa Scale (online supplemental file 3) was determined to be the most appropriate instrument for quality assessment via a consideration of tools available in the Systematic Review Toolbox. ${ }^{37}$ Any discrepancies between quality ratings will be resolved through discussion with the lead author. The quality ratings for each study will be provided in a table in the online supplemental material and studies with scores deemed less than satisfactory will be discussed as part of the narrative analysis. We will not exclude any articles from analysis due to low-quality scores, but sensitivity analysis will be performed to determine any effect of including studies that score less than 'good' according to Agency for Health Research and Quality standards. ${ }^{38}$

Evidence of publication bias and small-study effects will be assessed using two methods (available in the Stata meta suite).$^{39}$ First, we will examine contour-enhanced funnel plots for asymmetry using the Egger regression test. ${ }^{40}$ Second, we will use trim-and-fill analysis to estimate effects sizes that are adjusted for publication bias. ${ }^{41}$

\section{Analysis}

Data that meet all inclusion criteria will first be summarised descriptively and then analysed statistically. Data analysis will be conducted using Airtable and Stata V.16. ${ }^{35} 39$

Following the format of the original review and drawing on the synthesis without meta-analysis $(\mathrm{SWiM})^{42}$ reporting items, a narrative synthesis of study characteristics and findings will be conducted. This will provide a description and rationale for the reporting of groups used in the synthesis such as study populations, outcomes, study designs, methods used to assess the certainty of the evidence and limitations of the review. Study characteristics will be presented in summary tables across key variables (including study design, year, setting, country; population characteristics; exposure measures including number of items and whether validated; definition of racism used). If meta-analysis is not possible, the nature of the relationship between exposure and outcome (positive, negative or null) across key study characteristics will be summarised, following the approach in the original review. ${ }^{1}$

Although meta-analysis is planned, this will only become apparent when extracted data are reviewed for feasibility. If data are available, we will conduct analyses of associations between racism and health for different health outcome measures and at different time points. If possible, we will use random effects models to aggregate effect sizes. Subgroup analyses will be conducted for age, gender and ethnicity if possible. To assess the heterogeneity of studies, we will use the Q-statistic test and the $\mathrm{I}^{2}$ statistic. If the test for heterogeneity denoted as $\mathrm{I}^{2}$ (if $\mathrm{I}^{2}$ $\leq 25 \%$ ), studies will be considered homogeneous. Based on the Grading of Recommendations, Assessment, 
Development and Evaluation framework, ${ }^{43}$ we will rate the quality of the overall evidence across each outcome to conclude.

\section{Patient and public involvement}

No patient involvement.

\section{DISCUSSION}

As this is an updated systematic review and meta-analysis, we expect that while there has been a significant amount of recent research conducted in this space, we do not anticipate our findings to be vastly different from our original review. This review and meta-analysis will incorporate studies with participants from all ethnic/racial/cultural backgrounds and studies will not be limited to any one country or geographic area, and in doing so, we anticipate to show that this is a problem faced by not just one specific population but by children globally. That is, we expect the review to show that racism and racial discrimination negatively impact multiple health outcomes in children and youth from different ethnic/racial/cultural backgrounds and across contexts. We expect an increase of research in outcomes not considered in the original review, including sleep and inflammatory and immune biomarkers, as well as markers of epigenetic risk and cellular ageing and of endocrine and hormonal function. Increased attention on younger age groups, vicarious as well as direct exposure, longitudinal associations and populations and settings outside of the USA are also anticipated.

Due to the expectant increase in research surrounding this topic, a key contribution of the current study is to conduct a meta-analysis, which was not able to be conducted before. We expect that through this metaanalysis, we will be able to show rigorous and robust evidence showing the relationship between experiences of racism and health and well-being outcomes for children and youth. As this is the first meta-analysis of these studies, it will provide an evidence base for future research exploring the effect of racism and child health, as well as for policy development and service delivery.

Review findings will provide essential information for future research and policy priorities and inform the development effective evidenced-based targets for interventions to ameliorate the harmful impacts of racism on child and youth health.

\section{ETHICS AND DISSEMINATION}

Ethics approval is not required as this is a review of existing empirical findings and no primary data will be collected throughout the research.

The results from this review will be disseminated in peer-review publications and conference presentations as well as communicated more broadly through factsheets and summaries disseminated through academic institution press release and policy and practice partners.
Specific dissemination strategies will be codesigned with policy, practice and community stakeholders towards completion of the review.

Author affiliations

${ }^{1}$ Centre for Social Research and Methods, Australian National University, Canberra, Australian Capital Territory, Australia

${ }^{2}$ Population Health, Murdoch Childrens Research Institute, Parkville, Victoria, Australia

${ }^{3}$ School of Nursing and Midwifery, Monash University, Clayton, Victoria, Australia ${ }^{4}$ Department of Pediatrics, The University of Melbourne, Melbourne, Victoria, Australia

${ }^{5}$ Lee Kuan Yew Centre for Innovative Cities, Singapore University of Technology and Design, Singapore

${ }^{6}$ School of Sociology, Politics and International Studies, University of Bristol, Bristol, UK

${ }^{7}$ Epidemiology and Public Health, University College London, London, UK ${ }^{8}$ School of Humanities and Social Science, Deakin University, Burwood, Victoria, Australia

Twitter Mandy Truong @mandy_tru and Brigid Trenerry @BrigidTrenerry

Contributors NP conceptualised the review, contributed to all aspects of the protocol and the guarantor for the review. KD codrafted the protocol, developed the search strategy with medical librarians. RP and SG assisted with drafting and revising the protocol. MT, BT, SK, YK and YP reviewed the protocol draft.

Funding NP was supported by a National Health and Medical Research Council Career Development Fellowship (APP1123677).

Disclaimer The funding sources had no role in the design and conduct of the study; collection, management, analysis and interpretation of the data; preparation, review or approval of the manuscript and decision to submit the manuscript for publication.

Competing interests None declared.

Patient consent for publication Not required.

Provenance and peer review Not commissioned; externally peer reviewed.

Supplemental material This content has been supplied by the author(s). It has not been vetted by BMJ Publishing Group Limited (BMJ) and may not have been peer-reviewed. Any opinions or recommendations discussed are solely those of the author(s) and are not endorsed by BMJ. BMJ disclaims all liability and responsibility arising from any reliance placed on the content. Where the content includes any translated material, BMJ does not warrant the accuracy and reliability of the translations (including but not limited to local regulations, clinical guidelines, terminology, drug names and drug dosages), and is not responsible for any error and/or omissions arising from translation and adaptation or otherwise.

Open access This is an open access article distributed in accordance with the Creative Commons Attribution Non Commercial (CC BY-NC 4.0) license, which permits others to distribute, remix, adapt, build upon this work non-commercially, and license their derivative works on different terms, provided the original work is properly cited, appropriate credit is given, any changes made indicated, and the use is non-commercial. See: http://creativecommons.org/licenses/by-nc/4.0/.

\section{ORCID iDs}

Naomi Priest http://orcid.org/0000-0002-2246-0644

Saffron Karlsen http://orcid.org/0000-0003-0503-5088

Yvonne Kelly http://orcid.org/0000-0002-2936-3994

\section{REFERENCES}

1 Priest N, Paradies Y, Trenerry B, et al. A systematic review of studies examining the relationship between reported racism and health and wellbeing for children and young people. Soc Sci Med 2013;95:115-27.

2 Trent M, Dooley DG, Dougé J, et al. The impact of racism on child and adolescent health. Pediatrics 2019;144:e20191765.

3 Fuentes A, Ackermann RR, Athreya S, et al. AAPA statement on race and racism. Am J Phys Anthropol 2019;169:569-70.

4 Bonilla-Silva E. Rethinking racism: toward a structural interpretation. Am Sociol Rev 1997;62:465-80. 
5 Williams DR. Race and health: basic questions, emerging directions. Ann Epidemiol 1997;7:322-33.

6 Williams DR, Williams-Morris R. Racism and mental health: the African American experience. Ethn Health 2000;5:243-68.

7 Krieger N. Discrimination and Health inequities. In: Berkman L, Kawachi I, Glymour MM, eds. Social epidemiology. 2 edn. New York: Oxford University Press, 2014.

8 Bailey ZD, Krieger N, Agénor M, et al. Structural racism and health inequities in the USA: evidence and interventions. Lancet 2017;389:1453-63.

9 Heard-Garris NJ, Cale M, Camaj L, et al. Transmitting trauma: a systematic review of vicarious racism and child health. Soc Sci Med 2018;199:230-40.

10 Harrell SP. A multidimensional conceptualization of racism-related stress: implications for the well-being of people of color. Am J Orthopsychiatry 2000;70:42-57.

11 García Coll C, Lamberty G, Jenkins R, Crnic K, et al. An integrative model for the study of developmental competencies in minority children. Child Dev 1996;67:1891-914.

12 Paradies Y, Ben J, Denson N, et al. Racism as a determinant of health: a systematic review and meta-analysis. PLoS One 2015;10:e0138511.

13 Lewis TT, Cogburn CD, Williams DR. Self-reported experiences of discrimination and health: scientific advances, ongoing controversies, and emerging issues. Annu Rev Clin Psychol 2015;11:407-40.

14 Goosby BJ, Malone S, Richardson EA, et al. Perceived discrimination and markers of cardiovascular risk among low-income African American youth. Am J Hum Biol 2015;27:546-52.

15 Priest N, Chong S, Truong M, et al. Racial discrimination and socioemotional and sleep problems in a cross-sectional survey of Australian school students. Arch Dis Child 2020;105:1079-85.

16 Yip T. The effects of ethnic/racial discrimination and sleep quality on depressive symptoms and self-esteem trajectories among diverse adolescents. J Youth Adolesc 2015;44:419-30.

17 Yip T, Cheon YM, Wang Y, et al. Racial disparities in sleep: associations with discrimination among Ethnic/Racial minority adolescents. Child Dev 2020;91:914-31.

18 Garner P, Hopewell S, Chandler J, et al. When and how to update systematic reviews: consensus and checklist. BMJ 2016;354:i3507.

19 Moher D, Liberati A, Tetzlaff $\mathrm{J}$, et al. Preferred reporting items for systematic reviews and meta-analyses: the PRISMA statement. PLOS Med 2009;6:e1000097.

20 Shamseer L, Moher D. Preferred reporting items for systematic review and meta-analysis protocols (PRISMA-P) 2015: elaboration and explanation. BMJ 2016;354:i4086.

21 Higgins J, Green S, eds. Cochrane handbook for systematic reviews of interventions 5.1.0, 2011.

22 Sawyer SM, Afifi RA, Bearinger LH, et al. Adolescence: a foundation for future health. Lancet 2012;379:1630-40.

23 United Nations. Youth: United nations, 2020. Available: https://www. un.org/en/sections/issues-depth/youth-0/index.html2020

24 Patton GC, Sawyer SM, Santelli JS, et al. Our future: a Lancet Commission on adolescent health and wellbeing. Lancet 2016;387:2423-78.
25 Joshi KY. The Racialization of Hinduism, Islam, and Sikhism in the United States. Equity Excell Educ 2006;39:211-26.

26 Ysseldyk R, Talebi M, Matheson K, et al. Religious and ethnic discrimination: differential implications for social support engagement, civic involvement, and political consciousness. J Soc Polit Psych 2014;2:347-76.

27 Priest N, Ferdinand A. Mental health impacts of racism and attitudes towards diversity in Victorian schools. A summary of survey findings, 2014.

28 Priest N, Kavanagh A, Bécares L, et al. Cumulative effects of bullying and racial discrimination on adolescent health in Australia. $J$ Health Soc Behav 2019;60:344-61.

29 Priest N, Truong M, Chong S, et al. Experiences of racial discrimination and cardiometabolic risk among Australian children. Brain Behav Immun 2020;87:660-5.

30 Pearce J, Rafiq S, Simpson J, et al. Perceived discrimination and psychosis: a systematic review of the literature. Soc Psychiatry Psychiatr Epidemiol 2019;54:1023-44.

31 Elias A, Paradies Y. Estimating the mental health costs of racial discrimination. BMC Public Health 2016;16:1205.

32 O'Connor M, Sanson A, Hawkins MT, et al. Differentiating three conceptualisations of the relationship between positive development and psychopathology during the transition to adulthood. J Adolesc 2011;34:475-84.

33 Clarivate Analytics. EndNote reference management software [computer program]. Version X9. Philadelphia, USA, 2019.

34 Covidence. Coidence- better systematic review management Melbourne, Australia: Covidence, 2015. Available: https://www. covidence.org/2020

35 Liu H, Ofstad A. Airtable San Francisco, California, United States of America: Howie Liu, Andrew Ofstad, Emmett Nicholas, 2012. Available: https://airtable.com/

36 Wells GA, Tugwell P. The Newcastle-Ottawa scale (NOS) for assessing the quality of nonrandomized studies in meta-analyses 2019, 2020. Available: http://www.ohri.ca/programs/clinical_ epidemiology/oxford.asp

37 Marshall C. Systematic review toolbox, 2015. Available: http://syst ematicreviewtools.com/index.php

38 Viswanathan M, Ansari MT. Assessing the risk of bias of individual studies in systematic reviews of health care interventions. methods guide for effectiveness and comparative effectiveness reviews. Rockville (MD): Agency for Healthcare Research and Quality (US), 2008

39 StataCorp. Stata statistical software: release 16. College Station, TX: Stata Corp LP, 2019.

40 Sterne JA, Egger M. Addressing Reporting Biases. In: Cochrane handbook for systematic reviews of interventions, 2008: 297-333.

41 Duval S. A nonparametric "trim and fill" method of accounting for publication bias in meta-analysis. J Am Stat Assoc 2000;95:89-98.

42 Campbell M, McKenzie JE, Sowden A, et al. Synthesis without meta-analysis (swim) in systematic reviews: reporting guideline. $B M J$ 2020;368:16890.

43 Atkins D, Best D, Briss PA, et al. Grading quality of evidence and strength of recommendations. BMJ 2004;328:1490. 